\title{
Late results of the Royal Free Hospital prospective controlled trial of prednisolone therapy in hepatitis B surface antigen negative chronic active hepatitis
}

\author{
A P KIRK, S JAIN, S POCOCK, H C THOMAS, AND SHEILA SHERLOCK \\ From the Departments of Medicine and Clinical Epidemiology, Royal Free Hospital, London
}

SUMmARY A long-term follow-up of at least 10 years or until death of 44 patients taking part in a controlled prospective trial of prednisolone therapy in hepatitis B antigen negative chronic active hepatitis (lupoid hepatitis) has been performed at the Royal Free Hospital, London. Patients presenting between 1963 and 1967 were randomly allocated into control and treatment groups. Ten year life table survival curves showed a significantly improved survival in the treatment group where $63 \%$ of patients were alive at 10 years compared with only $27 \%$ in the control group (log rank test, $\mathrm{P}=0.03$ ). The median survival in the treatment group was 12.2 years compared with 3.3 years in the control group. The mean duration of treatment was 4.5 years. Age, presence of antinuclear factor, cirrhosis, or level of serum transaminases at presentation did not appear to affect survival. Male patients if untreated had a poorer prognosis than females $(P=0.02)$. The natural history of chronic active hepatitis appeared from clinical, biochemical, and histological findings to be from an active hepatitis or cirrhosis to inactive macronodular cirrhosis. Prednisolone therapy significantly improved survival by reducing mortality in the early active phase of the disease.

Chronic active hepatitis is defined as inflammation of the liver continuing without improvement for at least six months (Anthony et al., 1978), and with the histological findings of round cell infiltration of the portal tracts with moderate or severe piecemeal necrosis of liver cells extending outward into the parenchyma of the limiting plate. The biochemical features include raised serum transaminases and globulin levels. Various aetiological agents have been found to give this clinical and pathological picture, including the hepatitis B virus (Sherlock et al., 1970), drugs such as methyldopa (Eliastem and Holmes, 1971), and Wilson's disease (Scott et al., 1978). In many patients, however, such extrinsic agents or inherited defects cannot be found, and organ specific autoantibodies have been suggested as the aetiological factor. This form of the disease is more common in females than in males, and is often multisystemic, being associated with arthralgias (Bearn et al., 1956), thyroiditis (Read et al., 1963), Coombs' positive haemolytic anaemia (Mistilis and Blackburn, 1970), and ulcerative colitis (Holdsworth et al., 1965). It has been termed 'lupoid' hepatitis.

This disease rapidly progresses to early death from

Received for publication 2 August 1979 hepatocellular failure (Mackay and Wood, 1962). The few patients surviving the early years of active liver disease progress to macronodular cirrhosis and die from its complications (Read et al., 1963). Results of three controlled trials (Cook et al., 1971; Soloway et al., 1972; Murray-Lyon et al., 1973; Wright et al., 1977) showed that corticosteroid therapy considerably reduces the mortality in the early 'active' phase and this treatment is now generally accepted. In order to evaluate the longterm effects of steroid therapy on the course of the disease, and the number of years for which it is required, we have followed the patients initially reported by Cook et al., (1971) for a period of 10 to 15 years from diagnosis.

\section{Methods}

\section{PATIENTS}

A controlled prospective trial of prednisolone therapy in chronic active hepatitis was started at the Royal Free Hospital in 1963, and the results were reported in 1971 (Cook et al., 1971). The patients entered were all those admitted to hospital between 1963 and 1967, and had not received corticosteroid therapy. The diagnosis was based on the clinical and biochemical 
features of a hepatocellular illness of more than six months' duration where no other precipitating factor was elucidated and on the liver histology, which was available in 44 of the original 49 patients.

Patients were randomly divided into control and steroid treated groups by means of sealed envelopes. Those treated with steroids were started on prednisolone $15 \mathrm{mg}$ daily, but those in the control group did not receive a placebo, as it was felt essential to identify those patients receiving corticosteroids. Treatment was continued until the patients were asymptomatic and the liver function tests had returned towards normal; the duration varied considerably between patients. The two groups were reasonably well matched.

These patients have now been reviewed 10 to 15 years from entry into the trial. Five of the 49 patients initially reported have been excluded, as additional clinical and histologica evidence has made the diagnosis of chronic active hepatitis untenable, other factors such as alcohol being identified. Thirty of the remaining 44 patients have been seen at the Royal Free Hospital at six monthly or yearly intervals until at least 10 years from diagnosis, or until they died. Another 13 patients were traced through their general practitioners or referring hospitals. One patient was lost to follow-up five years after diagnosis. Information was sought on the clinical state, including therapy, the b ochemical findings, and, where possible, liver histology. In the 24 patients who died, details of the mode of death were available in 21 , and the post mortem findings in eight.

Determination of the presence of the hepatitis B surface antigen was not performed before 1970, and thus, in patients dying before this, hepatitis $B$ virus associated chronic active hepatitis cannot be excluded. The majority of these patients, however, were female, and all except one (in the treatment group) were British and therefore unlikely to be carrying the virus. None of the 21 patients tested for the hepatitis B surface antigen was positive.

\section{Results}

Patients were entered into the trial between 1963 and 1967 , allowing at least 10 years' follow-up in all but one case. Corticosteroids were given for periods ranging between six months and 13 years (mean 4.5 years), the duration being determined by the clinical and laboratory findings (Fig. 1). Two of the control patients underwent relapses which were treated with prednisolone (Fig. 2).

Six of the original 22 control patients $(27 \%)$ survived 10 years (four never having received corticosteroids) compared with 13 of the initial 22 treated patients $(63 \%)$. One patient was lost to follow-up at five years (Table 1). The life table survival curves over this 10 year period are shown in Fig. 3. In the 10 to 15 year period of follow-up, three patients died in the corticosteroid treated group, three patients

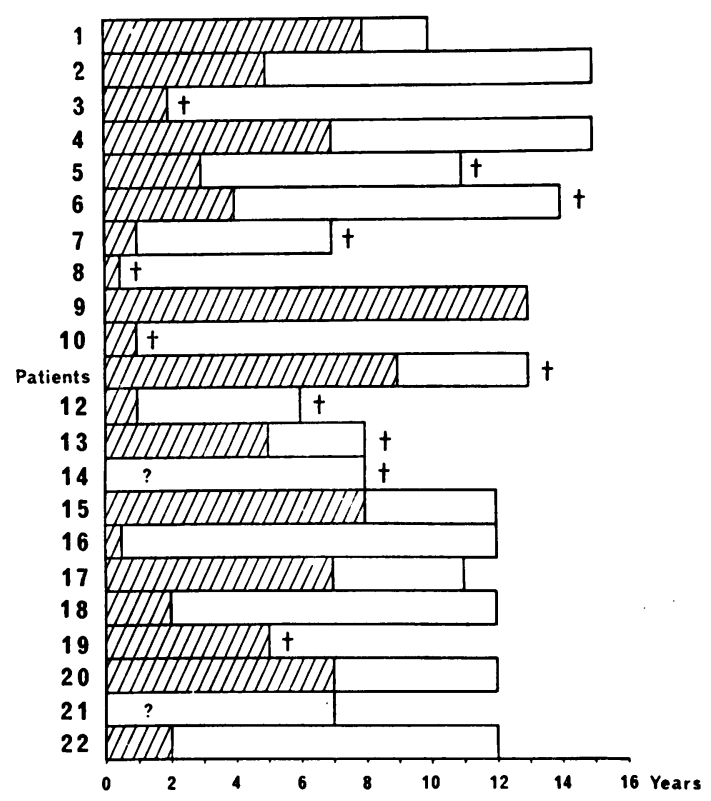

Fig. 1 Length of follow-up and duration of prednisolone therapy in prednisolone treated patients. $\mathbb{Z}$ Prednisolone therapy. † Death.

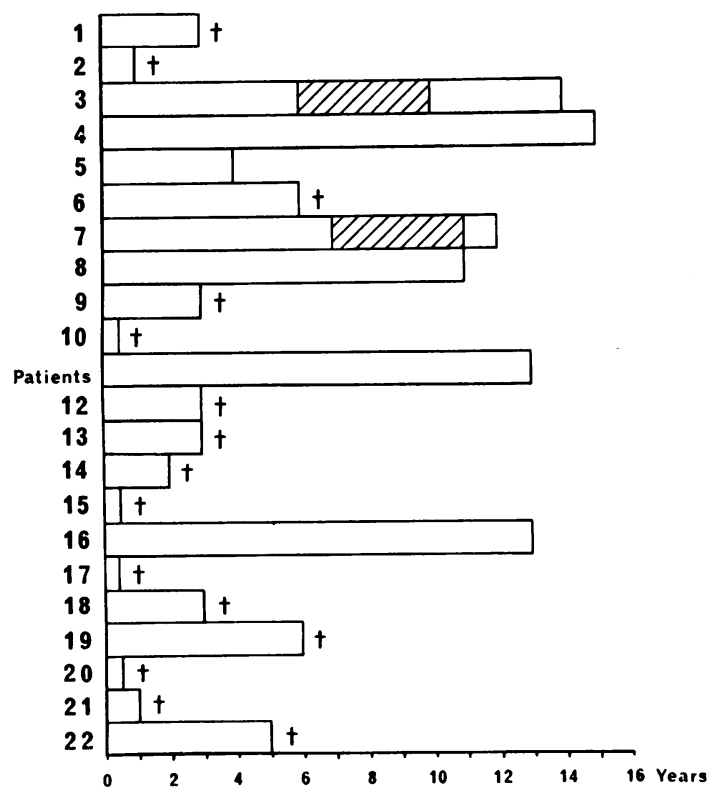

Fig. 2 Length of follow-up and prednisolone use in control patients. $\mathbb{Z}$ Prednisolone therapy. $\dagger$ Death. 
Table 1 Overall mortality at 10 years from entry into trial in control and prednisolone treated groups

\begin{tabular}{lcl}
\hline & Control & Prednisolone \\
\hline Total & 22 & 22 \\
Alive & 6 & 13 \\
Dead & 16 & 8 \\
Lost to follow-up & - & 1 \\
\hline
\end{tabular}

were lost to follow-up (two control and one prednisolone treated group), and the remainder have been followed for different periods. Incorporating this data, the log rank test for comparing the two life table survival curves shows that the effect of treatment in improving survival is statistically significant $(P=0.03)$. Estimated median survival times for the control and treatment groups are 3.3 years and $12 \cdot 2$ years respectively, although this last figure is not very precise because of incomplete follow-up.

Additional analyses using the life tables and log rank tests were performed to see whether age, sex,

Table 2 Age, sex, transaminase level, presence of antinuclear factor, and cirrhosis at presentation in survivors and non-survivors at 10 years in both control and prednisolone treated groups

\begin{tabular}{|c|c|c|c|c|}
\hline & \multicolumn{2}{|l|}{ Control } & \multicolumn{2}{|c|}{ Prednisolone } \\
\hline & Alive & Dead & Alive & Dead \\
\hline \multicolumn{5}{|l|}{ Age (yr) } \\
\hline Range & $14-53$ & $12-64$ & $3 \frac{1}{2}-62$ & $13-65$ \\
\hline Mean & 40 & 41 & $39^{2}$ & 34 \\
\hline Sex-M:F & $0: 6$ & $6: 10$ & $2: 11$ & $1: 7$ \\
\hline \multicolumn{5}{|l|}{ AST } \\
\hline Range & $19-245$ & $21-470$ & $32-470$ & $24-1000$ \\
\hline Median & 56 & 88 & 134 & 88 \\
\hline ANF & $5 / 6$ & $8 / 16$ & $6 / 13$ & $3 / 8$ \\
\hline Cirrhosis & $3 / 6$ & $12 / 16$ & $9 / 12$ & $6 / 9$ \\
\hline
\end{tabular}

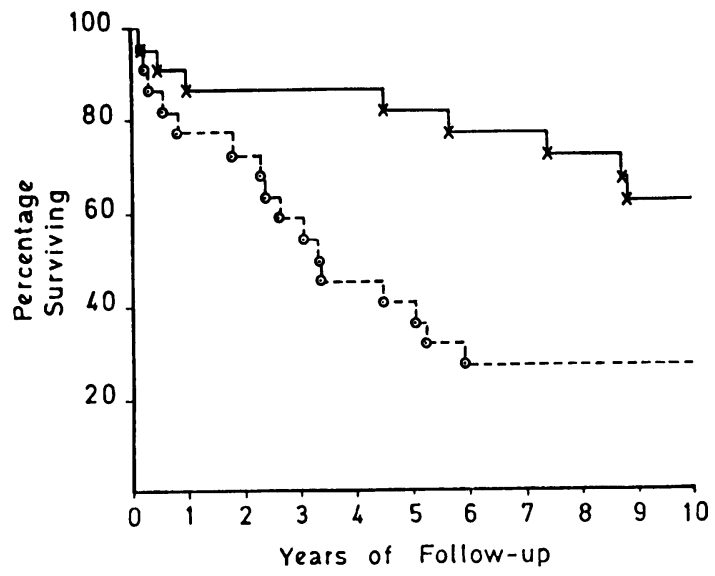

Fig. 3 Life table survival curves of control and prednisolone treated pationts. $\odot---\odot$ Controls. $\longleftrightarrow \longrightarrow$ Prednisolone.
Table 3 Time of death in control and prednisolone treated patients

\begin{tabular}{lcc}
\hline & \multicolumn{2}{l}{ Time of death } \\
\cline { 2 - 3 } & $0-5$ yr & $6-10$ yr \\
\hline Deaths & 14 & 2 \\
$\quad$ Control & 4 & 4 \\
Prednisolone & & \\
\hline
\end{tabular}

serum transaminase level, presence of serum antinuclear factor, or of cirrhosis at diagnosis had any association with survival (Table 2). Numbers are too small for definite conclusions, but there is some indication that the six untreated male patients had a poorer survival than the 16 untreated females (log rank test, $P=0.02$ ). With only three males in the treatment group it is not possible to say whether treated males fared worse than treated females. Age, antinuclear factor, serum transaminase level, and presence of cirrhosis at diagnosis had no effect on survival. Serum smooth muscle antibody estimations were not available at the outset of the trial, and thus cannot be assessed as a prognostic feature.

The time and mode of death were examined in patients not surviving 10 years. Fourteen of the 16 control patients who died in this period did so within five years of diagnosis. This tendency to early death was not evident in the prednisolone treated patients, where the eight deaths were spread evenly through the 10 year period (Table 3 ). The causes of death in the early (first five years) and the late stage (six to 10 years) and in those dying after 10 years are shown in Table 4. Hepatocellular failure was the mode of early (first five years) death in five of 14 untreated patients, but in none in the treatment group. One treated patient died later from liver failure. Fatal haemorrhage from gastro-oesophageal varices happened at any period in both groups. Of the six patients in the treatment group who died more than two years after prednisolone therapy ended, three died from

Table 4 Causes of death in control and prednisolone treated patients

\begin{tabular}{|c|c|c|c|}
\hline \multirow[t]{2}{*}{ Deaths } & \multicolumn{3}{|c|}{ Years } \\
\hline & $0-5$ & $6-10$ & $>10$ \\
\hline \multicolumn{4}{|l|}{ Control } \\
\hline GI bleed & 5 & 1 & - \\
\hline Hepatocellular failure & 5 & - & - \\
\hline Carcinoma & 3* & - & - \\
\hline Unknown & 1 & 1 & - \\
\hline Other & - & - & - \\
\hline \multicolumn{4}{|l|}{ Prednisolone } \\
\hline GI bleed & 4 & 1 & 2 \\
\hline Hepatocellular failure & - & 1 & - \\
\hline Carcinoma & - & $1+$ & - \\
\hline Unknown & - & - & - \\
\hline Other & - & - & $1 \neq$ \\
\hline
\end{tabular}

*Astrocytoma, carcinoma cervix and stomach †Carcinoma of colon $\ddagger$ Transplant 
bleeding oesophageal varices with associated hepatic failure, one from hepatocellular failure, one after liver transplant, and in one case the cause of death was not ascertained. Four patients, three untreated and one treated, died from unrelated carcinomas of

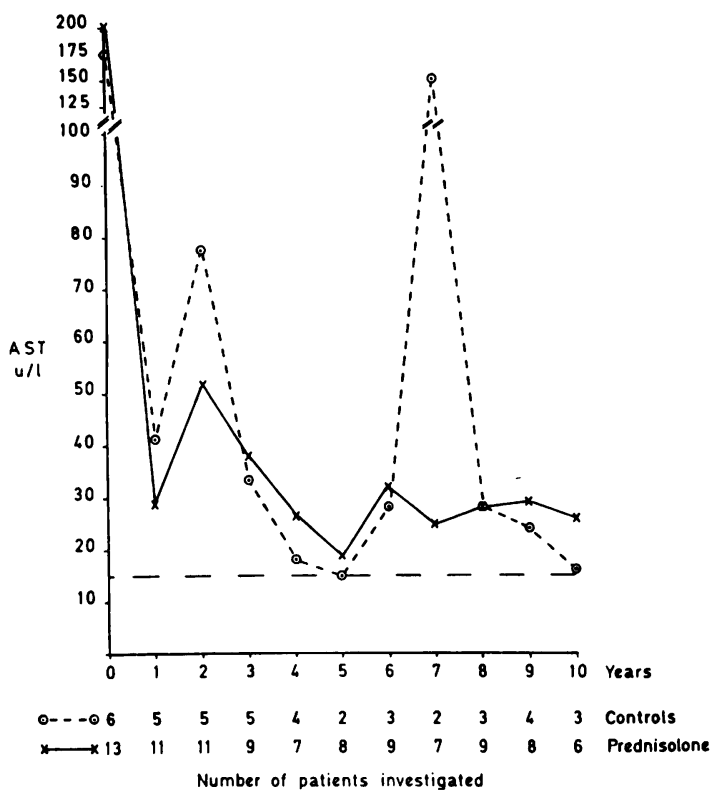

Fig. 4 Mean values of aspartate transaminase at yearly intervals in those patients surviving 10 years in the control and prednisolone treated groups.

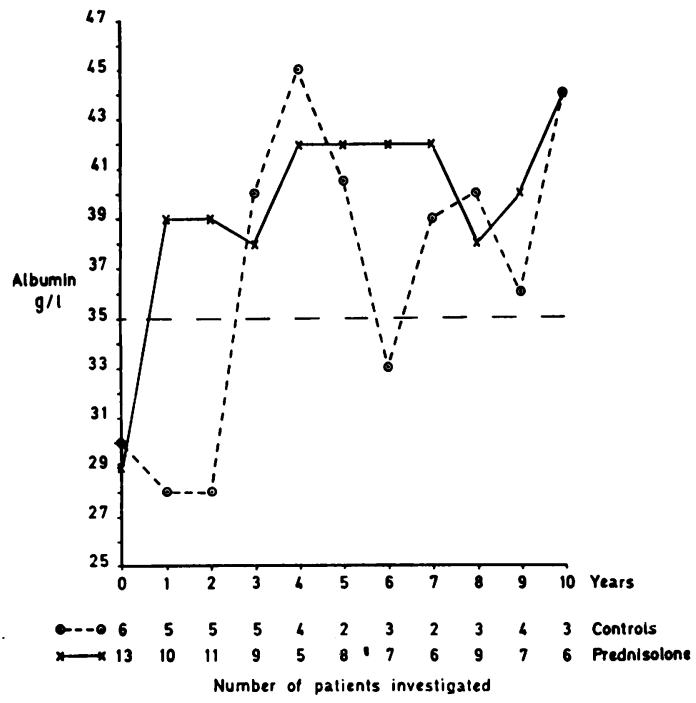

Fig. 5 Mean values for serum albumin at yearly intervals in those patients surviving 10 years in the control and prednisolone treated groups. stomach, cervix, colon, and brain.

Study of those surviving 10 years gives the natural history of non-fatal chronic active hepatitis. The mean values of serum aspartate transaminase in the six control and 13 treated patients showed a fall over the first four years and then reached a plateau between normal and twice normal (Fig. 4). Two of the control patients relapsed at six and seven years respectively, accounting for the marked rise in serum transaminase level. Both were subsequently treated with prednisolone and remitted. Prednisolone treated patients within one year showed a return of the serum albumin level to normal. In the control group normal values were reached only after three years (Fig. 5). Serum albumin levels fell in the two control patients who relapsed but returned to normal on treatment. These biochemical results suggest a trend towards well-compensated liver disease, and also suggest that this develops earlier in those patients receiving prednisolone.

Follow-up of liver histology was extremely limited and of limited value in view.of the sampling error in small biopsies. The pathological changes in the 10 year survivors in both the control (one patient) and the treatment groups (four patients) and the followup and post mortem results in the non-survivors (11 patients) suggest that, in keeping with the changes in liver function tests, the disease progresses from active hepatitis with or without cirrhosis to an inactive macronodular cirrhosis.

These conclusions are, at least, borne out by the clinical condition of the 10 year survivors. Six of these are no longer available for assessment, three having died and three being lost to follow-up, but all the remaining 13 patients (four control and nine treatment group) have clinically inactive liver disease. Two are troubled by mild ulcerative colitis, one has developed osteoporosis, one mild chronic pancreatitis, and one patient has become depressed.

\section{Discussion}

This controlled trial lasting more than 10 years allows a long-term view of a well-defined group of patients with hepatitis $B$ antigen negative chronic active hepatitis. From the control group the natural history of chronic active liver disease can be seen in a way that is no longer possible, as it would now be unethical to withhold corticosteroid therapy. The beneficial effect of corticosteroids was statistically significant on overall survival, and was most evident in the first five years from diagnosis. Later there was little effect in increasing survival. In the first five years in the untreated group deaths usually resulted from hepatocellular failure or gastrointestinal 
bleeding. After five years fatal hepatocellular failure was uncommon.

Corticosteroids are immunosuppressive agents (Williams and Granger, 1969; Thompson and Van Furth, 1970; Claman, 1972; Atkinson and Frank, 1973) and so may reduce the early mortality of chronic active hepatitis. If the immune response to either native or altered self antigens results in the hepatocellular dysfunction (Meyer zum Buschenfelde and Hopf, 1974), this would be reduced and thus hepatocellular destruction decreased. Corticosteroids also stimulate protein synthesis (Cain et al., 1970; Rothschild et al., 1975) and this may contribute to the beneficial response. Reduced liver cell destruction and enhanced hepatic protein synthesis may lead to the increased plasma albumin seen during the early period of the study.

In both control and treated patients clinical observation, liver function tests, and liver histology suggest a progression from active hepatitis or active cirrhosis to inactive macronodular cirrhosis. The degree of activity may fluctuate and clinical, biochemical, and presumably histological relapses may develop after as long as six or seven years of quiescence. This is well illustrated by the two patients in the control group in whom corticosteroid therapy had to be given. From the four untreated patients, all with histologically proven chronic active hepatitis, who survived more than 10 years, the self-limiting nature of the disease is seen. In the absence of therapeutic immunosuppression this may, in part, be related to the state of immunodeficiency that has been documented in patients with chronic liver disease (Thomas, 1977). This 'immune' deficiency might also account for the $9 \%$ of patients who developed unrelated neoplasms-14\% in the control group and $5 \%$ in the treatment group; this compares with a rate of $1.3 \%$ in the general population (Office of Population Censuses and Surveys, 1971).

Age, presence of serum antinuclear factor, high serum transaminase levels, and an established cirrhosis were not of prognostic value. Untreated males had a higher mortality than females, but as the number of male patients was small and the majority were in the control group the significance of this observation is difficult to assess.

The sequence of clinical, biochemical, and histological changes in hepatitis B surface antigen negative chronic active hepatitis suggests a progression to inactive macronodular cirrhosis. This may be the result of the secondary immunodepression that develops in chronic liver disease (Thomas, 1977). If the patients survive the active phase, then long-term survival may be anticipated. Prednisolone therapy reduces mortality in the crucial early period. This study confirms the well-established fact that steroid therapy is mandatory, and suggests that the required duration of treatment is about five years.

\section{References}

Anthony, P. P., Ishak, K. G., Nayak, N. C., Poulson, H. E., Scheuer, P. T., and Sobin, L. H. (1978). Morphology of cirrhosis: recommendations on definition, nomenclature and classification by a working group sponsored by the World Health Organisation. Journal of Clinical Pathology, 31, 395-414.

Atkinson, J. P., and Frank, M. M. (1973). Effect of cortisone therapy on serum complement components. Journal of Immunology, 1113 1061-1066.

Bearn, A. G., Kunkel, H. G., and Slater, R. J. (1956). The problem of chronic liver disease in young women. American Journal of Medicine, 21, 3-15.

Cain, G. D., Mayer, G., and Jones, E. A. (1970). Augmentation of albumin synthesis but not fibrinogen synthesis by corticosteroids in patients with hepatocellular disease. Journal of Clinical Investigation, 49, 2198-2204.

Claman, H. M. (1972). Corticosteroids and lymphoid cells. New England Journal of Medicine, 287, 388-397.

Cook, G. C., Mulligan, R., and Sherlock, S. (1971). Controlled trial of corticosteroid therapy in chronic active hepatitis. Quarterly Journal of Medicine, 40, 159-185.

Eliastem, M., and Holmes, A. W. (1971). Hepatitis, arthritis and lupus cell phenomena caused by methyldopa. American Journal of Digestive Disease, 16, 1014-1018.

Holdsworth, C. D., Hall, E. W., Dawson, A. M., and Sherlock, S. (1965). Ulcerative colitis in chronic liver disease. Quarterly Journal of Medicine, 34, 211-227.

Mackay, I. R., and Wood, I. J. (1962). Lupoid hepatitis: a comparison of 22 cases with other types of chronic liver disease. Quarterly Journal of Medicine, 31, 485-507.

Meyer zum Buschenfelde, K. H., and Hopf, U. (1974). Studies on the pathogenesis of experimental chronic active hepatitis in rabbits. Induction of the disease and protective effect of allogenic liver specific proteins. British Journal of Experimental Pathology, 55, 498-513.

Mistilis, S. P., and Blackburn, C. R. B. (1970). Active chronic hepatitis. American Journal of Medicine, 48, 484-495.

Murray-Lyon, I. M., Stern, R. B., and Williams, R. (1973). Controlled trial of prednisolone and azathioprine in active chronic hepatitis. Lancet, 1, 735-737.

Office of Population Censuses and Surveys (1974). Morbidity Statistics for General Practice 1970-1971. HMSO; London.

Read, A. E., Sherlock, S., and Harrison, C. V. (1963). Juvenile cirrhosis part of a systemic disease - the effect of corticosteroid therapy. Gut, 4, 378-393.

Rothschild, M. A., Oratz, M., and Schreiber, ?. (1975), Regulation of albumin metabolism. Annual Review of Medicine, 26, 91-104.

Scott, J., Gollan, J. L., Samourian, S., and Sherlock, S. (1978). Wilson's disease presenting as chronic active hepatitis. Gastroenterology, 74, 645-651. 
Sherlock, S., Fox, R. A., Niazi, S. P., and Scheuer, P. J. Thompson, J., and van Furth, R. (1970). The effect of (1970). Chronic liver disease and primary liver cell carcinoma with hepatitis associated (Australia) antigen in serum. Lancet, 1, 1243-1256.

Soloway, R. D., Summerskill, W. H. J., Baggenstoss, A. H., Geall, M. G., Gitnick, G. L., Elveback, L. R., and Schoenfield, L. J. (1972). Clinical, biochemical and histological remission of severe chronic active liver disease: a controlled study of treatments and early prognosis. Gastroenterology, 63, 820-833.

Thomas, H. C. (1977). Immune response in hepatic cirrhosis. Animal and human studies. Proceedings of the Royal Society of Medicine, 70, 521-525.

glucocorticosteroids on the kinetics of mononuclear phagocytes. Journal of Experimental Medicine, 131, 429-442.

Williams, T. W., and Granger, G. A. (1969). Lymphocyte in vitro cytotoxicity, mechanism of lymphotoxin induced target cell destruction. Journal of Immunology, 102, 911-918.

Wright, E. C. Seeff, L. B., Berk, P. D., Jones, E. A., and Plotz, P. H. (1977). Treatment of chronic active hepatitis. An analysis of three controlled trials. Gastroenterology, 73, 1422-1430. 